\title{
HIGH RESOLUTION REMOTE SENSING IMAGE SEGMENTATION BASED ON GRAPH THEORY AND FRACTAL NET EVOLUTION APPROACH
}

\author{
Yi Yang, Haitao Li, Yanshun Han, Haiyan Gu \\ Key Laboratory of Geo-informatics of State Bureau of Surveying and Mapping, \\ Chinese Academy of Surveying and Mapping, \\ Beijing 100830, China \\ Yangyi@casm.ac.cn \\ lhtao@casm.ac.cn \\ hys_han@casm.ac.cn \\ guhy@casm.ac.cn
}

KEY WORDS: Image Segmentation, Graph Based, FNEA, Merging Criterion

\begin{abstract}
:
Image segmentation is the foundation of further object-oriented image analysis, understanding and recognition. It is one of the key technologies in high resolution remote sensing applications. In this paper, a new fast image segmentation algorithm for high resolution remote sensing imagery is proposed, which is based on graph theory and fractal net evolution approach (FNEA). Firstly, an image is modelled as a weighted undirected graph, where nodes correspond to pixels, and edges connect adjacent pixels. An initial object layer can be obtained efficiently from graph-based segmentation, which runs in time nearly linear in the number of image pixels. Then FNEA starts with the initial object layer and a pairwise merge of its neighbour object with the aim to minimize the resulting summed heterogeneity. Furthermore, according to the character of different features in high resolution remote sensing image, three different merging criterions for image objects based on spectral and spatial information are adopted. Finally, compared with the commercial remote sensing software eCognition, the experimental results demonstrate that the efficiency of the algorithm has significantly improved, and the result can maintain good feature boundaries.
\end{abstract}

\section{INTRODUCTION}

With the development of remote sensing technology and the improvement of satellite spatial resolution, high resolution remote sensing images are widely used in various fields. High resolution remote sensing images have clear details and rich spatial and texture information. In order to take full advantage of this information, we started paying attention to the objectoriented image analysis.

Object-oriented image analysis starts with the crucial initial step of grouping neighbouring pixels into meaningful areas, which can be handled by image segmentation. Generally image segmentation is defined as a process of splitting an image into regions based on some criteria (intensity, colour, texture, orientation energy). The goal of image segmentation is to simplify or change the representation of an image into something that is more meaningful and easier to analysis. The quality of segmentation result greatly impacts the precision of the following analysis, such as classification, understanding and recognition.

For the past decade, many scholars and institutions have begun research on image segmentation and form a series of sophisticated algorithm and techniques. High resolution remote sening image segmentation, which is different from traditional image segmentation, contains many objects of different size, but most segmentation algorithm specify a spatial sclae at the object. We should be able to describe objects in a hierarchical scale, as a result we apply the mutil-scale image segmentaiton.

\section{FRACTAL NET EVOLUTION APPROACH}

Fractal net evolution approach (FNEA), as a widely used multiscale segmentation algorithm, was first introduced by Baatz and Schäpe (2000), and played an important role in the commercial software eCognition. This algorithm quickly became one of the most important segmentation algorithms within the objectoriented image analysis domain.

The basic idea of the algorithm is a bottom-up region merging technique. It starts with each image pixel as a separate object. Subsequently, pairs of image objects are merged into large objects at each step (Figure 1). The process terminates when no pair of objects satisfies the merging criterion. We will talk about the merging criterion later.

FNEA rely a key control, called the scale parameter, which controls the internal heterogeneity of the image objects and is therefore correlated with their average size, i.e., a larger value of the scale parameter allows a higher internal heterogeneity, which increases the number of pixels per image object.

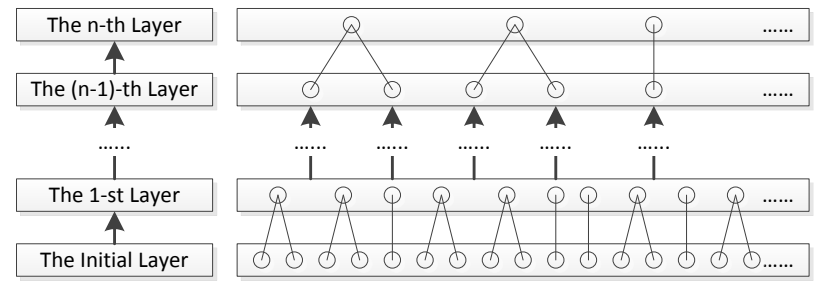

Figure 1. The object merging process of FNEA

However, the origial algorithm starts with the initial layer formed by image piexls, spends too much time on the first meging step, especially when we applied it to high resolution remote sensing image. An effectively improved method is using a fast segmentation algorithm to create the initial object layer. In this paper, we choose a graph based image segmentation algorithm, which runs in time nearly linear in the number of image pixels, to participate in our algorithm. The specific process is shown in Figure 2. 


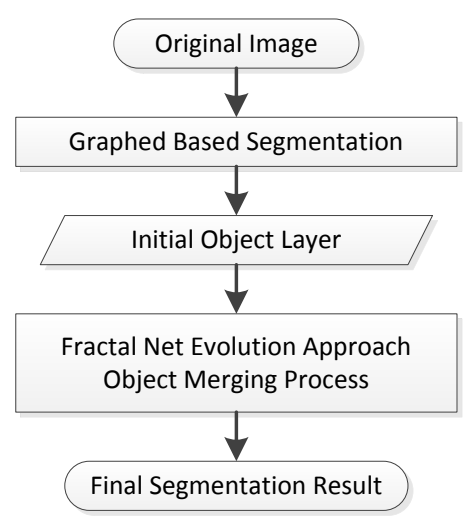

Figure 2. The process of our algorithm

An initial object layer can be obtained efficiently from graphbased segmentation. Then FNEA starts with the initial object layer and carries out the object merging process to get the final segmentation result.

\section{GRAPH BASED IMAGE SEGMENTATION}

Graph based image segmentation techniques generally represent the problem in terms of a weighted undirected graph $G=(V, E)$, where nodes correspond to pixels, and edges connect adjacent pixels. Each edge has a corresponding weight, which is a nonnegative measure of the dissimilarity between adjacent pixels. The graph is then partitioned according to a criterion designed to model "good" clusters. Each partition of the nodes output from these algorithms is considered an object segment in the image. Some popular algorithms of this category are minimum cut (Wu and Leahy 1993), average cut (Sarkar and Boyer 1996), normalize cut (Shi and Malik 2000, Kong et al. 2013), min-max cut (Ding et al. 2001), ratio cut (Wang et al. 2003) et al.

Felzenszwalb and Huttenlocher (2004) developed an efficient segmentation algorithm based on graph theory, and show that although this algorithm make greedy decisions it produces segmentation that satisfy global properties.

The internal difference of an object $C$ is defined as:

$$
\operatorname{Int}(C)=\max _{e \in M S T(C, E)} \omega(e)
$$

where $\omega(e)$ means the weight of edge $e$ in the minimum spanning tree.

Difference between two objects $\mathrm{C}_{1}, \mathrm{C}_{2}$ is defined as: $\operatorname{Dif}\left(\mathrm{C}_{1}, \mathrm{C}_{2}\right)=\min _{\mathrm{v}_{\mathrm{i}} \in \mathrm{C}_{1}, \mathrm{v}_{\mathrm{j}} \in \mathrm{C}_{2},\left(\mathrm{v}_{\mathrm{i}}, \mathrm{v}_{\mathrm{j}}\right) \in \mathrm{E}} \omega\left(\mathrm{v}_{\mathrm{i}}, \mathrm{v}_{\mathrm{j}}\right)$

where $\omega\left(v_{i}, v_{j}\right)$ means the weight of edge connecting $v_{i}$ and $v_{j}$.

Criteria for merging two objects $\mathrm{C}_{1}, \mathrm{C}_{2}$ is defined as:

$$
\begin{aligned}
& \mathrm{D}\left(\mathrm{C}_{1}, \mathrm{C}_{2}\right)=\left\{\begin{array}{c}
\text { true if } \operatorname{Dif}\left(\mathrm{C}_{1}, \mathrm{C}_{2}\right)>\operatorname{MInt}\left(\mathrm{C}_{1}, \mathrm{C}_{2}\right) \\
\text { false otherwise }
\end{array}\right. \\
& \operatorname{MInt}\left(\mathrm{C}_{1}, \mathrm{C}_{2}\right)=\min \left(\operatorname{Int}\left(\mathrm{C}_{1}\right)+\tau\left(\mathrm{C}_{1}\right), \operatorname{Int}\left(\mathrm{C}_{2}\right)+\tau\left(\mathrm{C}_{2}\right)\right)(4) \\
& \tau(\mathrm{C})=\mathrm{k} /|\mathrm{C}|
\end{aligned}
$$

where $|\mathrm{C}|$ denotes the size of object $\mathrm{C}$ and $\mathrm{k}$ is some constant parameter.

An image is modelled to a graph $G=(V, E)$, where are $n$ vertices and $\mathrm{m}$ edges. $S$ is the segmentation result, $C$ is some object in $S$. The segmentation algorithm produces segmentation as follows:

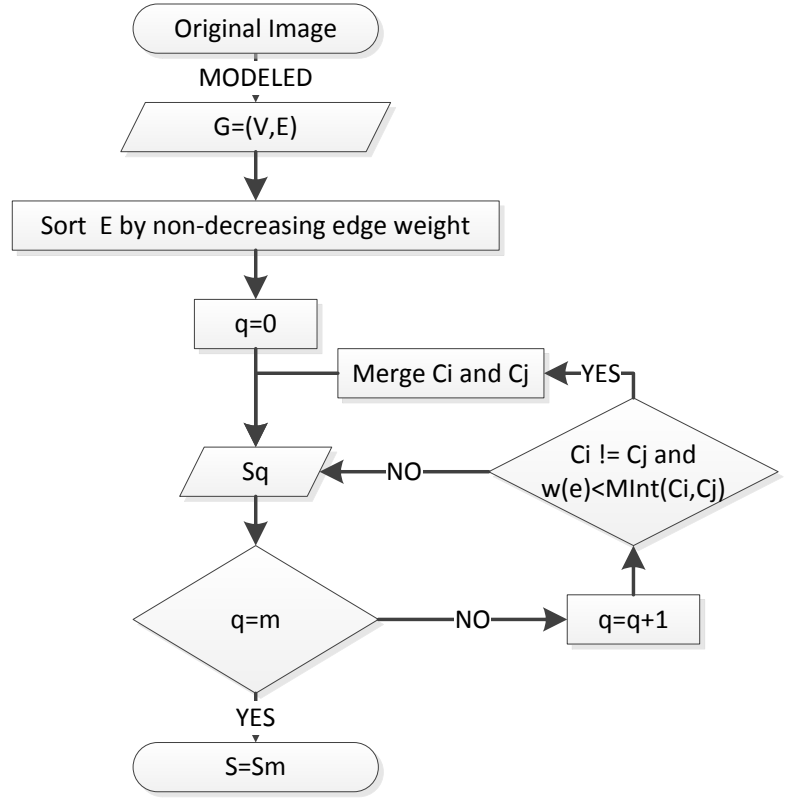

Figure 3. The process of graph-based segmentation

1) Sort $E$ into $\pi=\left(\mathrm{e}_{1}, \ldots, \mathrm{e}_{\mathrm{m}}\right)$, by non-decreasing edge weight.

2) Start with a segmentation $S^{0}$, where each vertex $v_{i}$ is in its own object.

3) Repeat step 4 for $q=1, \ldots, m$.

4) Construct $S^{q}$ given $S^{q-1}$ as follows. Let $v_{i}$ and $v_{j}$ denote the vertices connected by the q-th edge in the ordering, i.e., $\mathrm{e}_{\mathrm{q}}=\left(v_{i}, v_{j}\right)$. If $v_{i}$ and $v_{j}$ are in disjoint objects of $S^{q-1}$ and $\omega\left(\mathrm{e}_{\mathrm{q}}\right)$ is small compared to the internal difference of both those objects, then merge the two objects otherwise do nothing. More formally, let $C_{i}^{q-1}$ be the object of $S^{q-1}$ containing $v_{i}$ and $C_{j}^{q-1}$ the object containing $v_{j}$. If $C_{i}^{q-1} \neq C_{j}^{q-1}$ and $\omega\left(\mathrm{e}_{\mathrm{q}}\right)<\operatorname{MInt}\left(C_{i}^{q-1}, C_{j}^{q-1}\right)$ then $S^{q}$ is obtained from $S^{q-1}$ by merging $C_{i}^{q-1}$ and $C_{j}^{q-1}$. Otherwise $S^{q}=S^{q-1}$.

5) $\quad \operatorname{Return} S=S^{m}$.

\section{MERGING CRITERION}

The merging decision is based on a measure of the heterogeneity or some cost function. According to the character of different features in high resolution remote sensing image, we tried three different merging criterions for image objects based on spectral and spatial information.

\section{1 eCognition Criterion}

A merging cost, considered in eCognition, is described the change of heterogeneity in a virtual merge. $S^{\prime}$ would be the virtual merging object.

The increase of heterogeneity $f$ has to be less than a threshold:

$$
\mathrm{f}=\mathrm{w}_{\text {color }} \Delta \mathrm{h}_{\text {color }}+\mathrm{w}_{\text {shape }} \Delta \mathrm{h}_{\text {shape }}
$$

where $\mathrm{w}_{\text {color }}$ and $\mathrm{w}_{\text {shape }}$ are the weight of spectral and shape, $\mathrm{w}_{\text {color }}+\mathrm{w}_{\text {shape }}=1 . \Delta \mathrm{h}_{\text {color }}$ and $\Delta \mathrm{h}_{\text {shape }}$ are the increase of spectral and shape heterogeneity.

The increase of spectral heterogeneity $\Delta \mathrm{h}_{\text {color }}$ is defined as following:

$$
\Delta h_{\text {color }}=n^{\prime} \sigma^{\prime}-\left(n_{1} \sigma_{1}+n_{2} \sigma_{2}\right)
$$


where $\sigma$ and $\mathrm{n}$ are the standard deviation and size of object S, respectively.

The increase of shape heterogeneity $\Delta \mathrm{h}_{\text {shape }}$ is a value that describes the improvement of the shape with regard to smoothness and compactness of an object's shape. It is defined as following:

$$
\Delta \mathrm{h}_{\text {shape }}=\mathrm{w}_{\text {compact }} \Delta \mathrm{h}_{\text {compact }}+\mathrm{w}_{\text {smooth }} \Delta \mathrm{h}_{\text {smooth }}
$$

where $\mathrm{w}_{\text {compact }}$ is the weight of compactness, $\mathrm{w}_{\text {smooth }}$ is the weight of smoothness.

$$
\begin{aligned}
& \Delta \mathrm{h}_{\text {compact }}=\mathrm{n}^{\prime} \mathrm{l}^{\prime} / \sqrt{\mathrm{n}^{\prime}}-\left(\mathrm{n}_{1} \mathrm{l}_{1} / \sqrt{\mathrm{n}_{1}}+\mathrm{n}_{2} \mathrm{l}_{2} / \sqrt{\mathrm{n}_{2}}\right) \\
& \Delta \mathrm{h}_{\text {smooth }}=\mathrm{n}^{\prime} \mathrm{l}^{\prime} / \mathrm{b}^{\prime}-\left(\mathrm{n}_{1} \mathrm{l}_{1} / \mathrm{b}_{1}+\mathrm{n}_{2} \mathrm{l}_{2} / \mathrm{b}_{2}\right)
\end{aligned}
$$

where $\mathrm{l}$ is perimeter of object, $\mathrm{b}$ is perimeter of object's bounding box.

\subsection{Fisher Criterion}

Bilodeau et al. used a variant of Fisher's criterion to represent the heterogeneity between the adjacent objects, which is defined as follows:

$$
\mathrm{F}=\frac{\left|\mu_{1}-\mu_{2}\right|}{\sqrt{\sigma_{1}^{4}+\sigma_{2}^{4}}}
$$

where $\mu$ and $\sigma^{2}$ are the mean and variance of object $S$, respectively.

In order to merge objects of close similarity as well as with large intersection. So we use the merging score formula:

$$
\text { score }=\frac{\mid \mu_{1}-\mu_{2 \mid}}{\sqrt{\sigma_{1}^{4}+\sigma_{2}^{4}}} \times \frac{P}{L}
$$

where $\mathrm{P}$ is the perimeter of small object in $S_{1}$ and $S_{2}$, L is the common edge length of $S_{1}$ and $S_{2}$.

Given a object, we will find the smallest score among its neighbouring objects, and then merge the two obejcts if the score is less than a threshold and the common edge is not too small $(\mathrm{P} / \mathrm{L}<5)$.

\subsection{Full $\lambda$-Schedule Criterion}

Robinson et al. proposed a novel fast implementation of the full $\lambda$-schedule algorithm for segmentation. In this algorithm, the decision to merge objects $S_{1}$ and $S_{2}$ occurs when $t$ is less than a threshold where $t$ is given by

$$
t=\frac{\frac{n_{1} n_{2}}{n_{1}+n_{2}}\left(\mu_{1}-\mu_{2}\right)^{2}}{L}
$$

where $\mu$ and $n$ are the mean and size of object $S$, respectively, $\mathrm{L}$ is the common edge length.

\section{RESULTS AND ANALYSIS}

We choose four WorldView-2 images which covering a study area in Heilongjiang, China. They are named as Image1, Image2,
Image 3 and Image4 and exposed in Figures 4. The information of the Images are presented in Table 1.

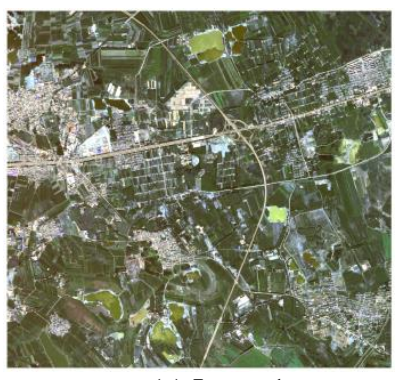

(a) Image 1

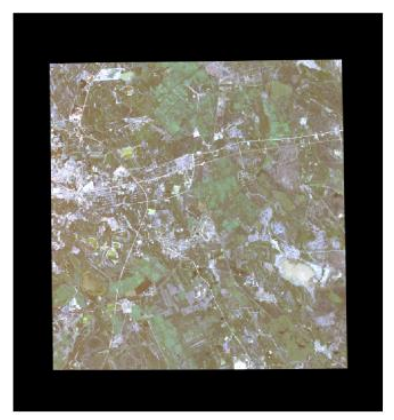

(c) Image 3

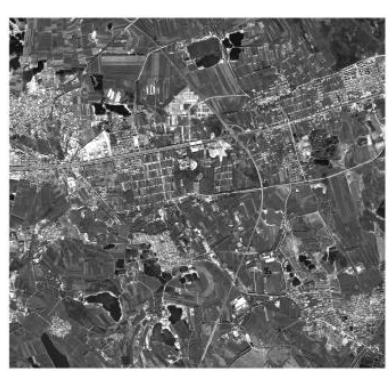

(b) Image2

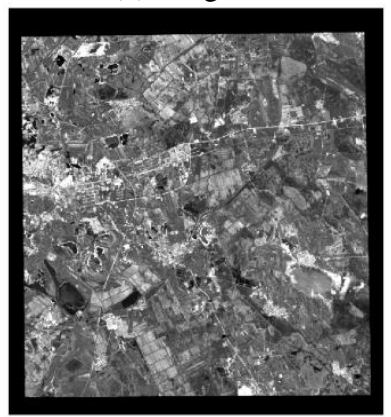

(d) Image4
Figure 4. Images used for experiments

\begin{tabular}{|c|c|c|c|c|}
\hline Name & Width & Height & Band & Size \\
\hline Image1 & 3836 & 3561 & 4 & $105 \mathrm{M}$ \\
\hline Image2 & 15341 & 14241 & 1 & $418 \mathrm{M}$ \\
\hline Image3 & 10656 & 11424 & 4 & $929 \mathrm{M}$ \\
\hline Image4 & 36704 & 39680 & 1 & $2.71 \mathrm{G}$ \\
\hline
\end{tabular}

Table 1. Images' information

The performance of the proposed algorithm has been evaluated using the four images in our application, compared with FNEA in eCognition. The overall execution times of all images are compared in table 2.

\begin{tabular}{|c|c|c|}
\hline Image & eCognition Time & Our Time \\
\hline Image1 & $1 \mathrm{~min}$ & $1 \mathrm{~min}$ \\
\hline Image2 & $20 \mathrm{~min}$ & $15 \mathrm{~min}$ \\
\hline Image3 & $12 \mathrm{~min}$ & $10 \mathrm{~min}$ \\
\hline Image4 & $135 \mathrm{~min}$ & $54 \mathrm{~min}$ \\
\hline
\end{tabular}

Table 2. Compared results of all images

As shown in this table, the speed of our algorithm is much better than eCognition, especially the size of image is large. When we use the image named Image 4 in eCognition, the tips show that it spent 70 minutes on the first cycle to create the initial object layer, which nearly half of the whole time. Obviously, using a fast segmentation algorithm to create the initial object layer is a correct choice of improving the efficiency of the algorithm.

The details of the segmentation result are compared in Figure 57. 


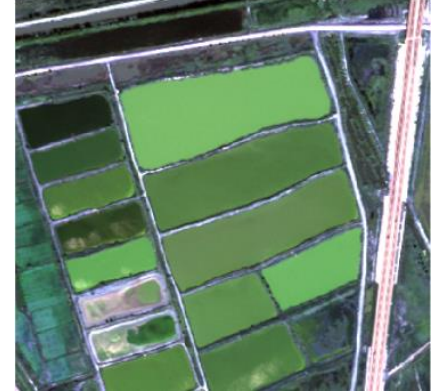

(a) Original image

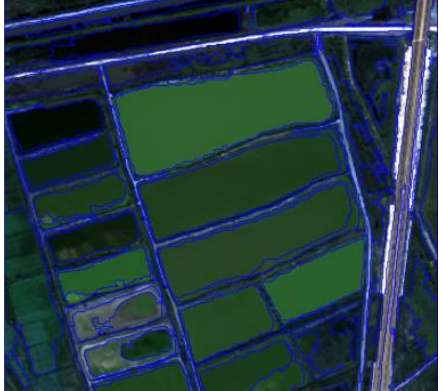

(b) Segmentation result of eCognition

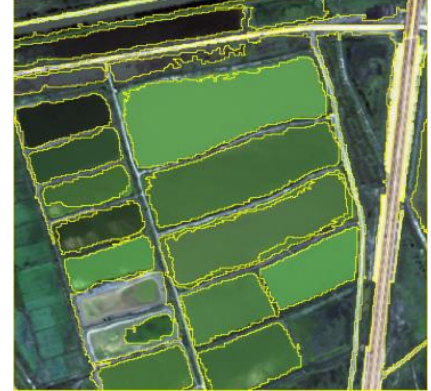

(c) Segmentation result of our algorithm

Figure 3. The segmentation result of vegetation

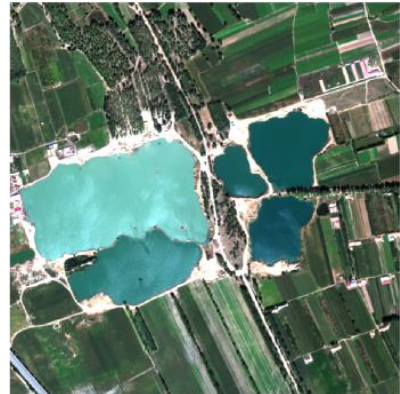

(a) Original image

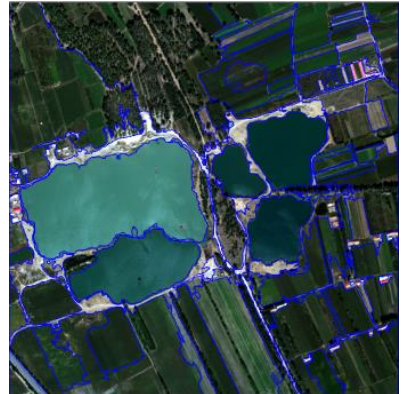

(b) Segmentation result of eCognition

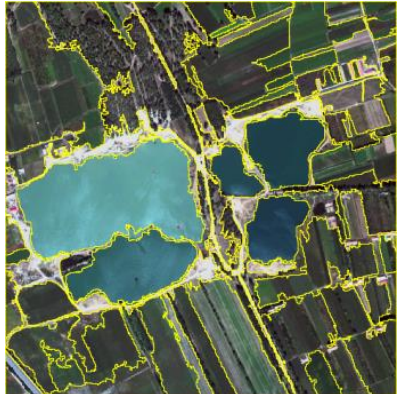

(c) Segmentation result of our algorithm Figure 6 . The segmentation result of water area

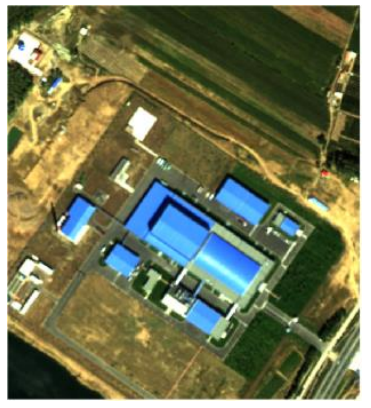

(a) Original image

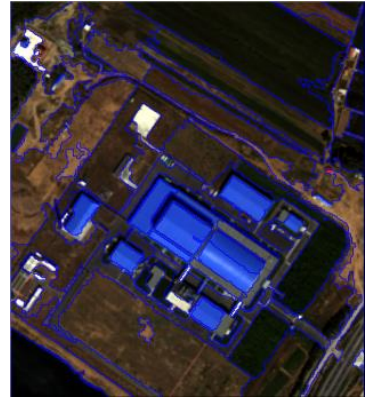

(b) Segmentation result of eCognition (c) Segmentation result of our algorithm Figure 7. The segmentation result of buildings
We choose several different features in high resolution remote sensing image. Figure 5-7 show the segmentation result of vegetation, water area and buildings, respectively. It can be noted that our algorithm can maintain good feature boundaries in main areas and the extracted contours are consisted with the real edge of objects in the image.

\section{SUMMARY AND CONCLUSIONS}

Image Segmentation for high resolution remote sensing image is a crucial and essential step for object oriented image analysis. In this paper we proposed an efficient algorithm combined FNEA with graphed based image segmentation. Moreover, three different merging criterions were introduced to compute the heterogeneity of objects.

From the experiment result, we found the algorithm is much faster than the original FNEA, which is integrated in the commercial software eCognition.

Future work should focus on two issues. The first is using parallel processing to speed up the segmentation algorithm. The second is taking into account the texture features to compute the heterogeneity.

\section{REFERENCES}

Morris O. J., Lee M. de J., Constantinides A. G., 1986. Graph theory for image analysis: an approach based on the shortest spanning tree. Communications Radar \& Signal Processing Iee Proceedings F, 133(2), pp.146-152.

Wu Z., Leahy R., 1993. An optimal graph theoretic approach to data clustering: theory and its application to image segmentation. IEEE transactions on pattern analysis and machine intelligence, 15(11), pp.1101-1113.

Shi J., Malik J., 2000. Normalized cuts and image segmentation. IEEE transactions on pattern analysis and machine intelligence, 22(8), pp.888-905.

Ding C., He X. F., Zha H. Y., Gu M., Simon H. D., 2001. A min-max cut algorithm for graph partiioning and data clustering. In proceedings of ICDM, pp.107-114.

Wang S., Siskind J. M., 2003. Image segmentation with ratio cut. IEEE transactions on pattern analysis and machine intelligence, 25(8), pp.676-690. 
Felzenszwalb P. F., Huttenlocher D. P., 2004. Efficient graphbased image segmentation. International Journal of Computer Vision, 59(2), pp.167-181.

Bilodeau G. A., Shu Y. Y., Cheriet F., 2006. Multistage graphbased segmentation of thoracoscopic images. Computerized Medical Imaging \& Graphics, 30(8), pp.437-446.

Baatz M., Schape A., 2000. Multiresolution Segmentation: an optimization approach for high quality multi-scale image segmentation. Angewandte Geographische Information Sverarbeitung, 12, pp.12-23.

Benz U. C., Hofmann P., Willhauck G., 2004. Multi-resolution object-oriented fuzzy analysis of remote sensing data for GISready information. Isprs Journal of Photogrammetry \& Remote Sensing, 58, pp.239-258.

Redding N. J., Crisp D. J., Tang D., 1999. An efficient algorithm for mumford-shah segmentation and its application to SAR imagery. Proc. Conf. Digital Image Computing Techniques and applications (DICTA), pp.35-41. 